\title{
Mathematical Modelling of Malaria Transmission in North Senatorial Zone of Taraba State Nigeria
}

\author{
${ }^{1}$ A. A. Momoh, ${ }^{2}$ A. Tahir, ${ }^{3}$ E. Omokhuale, ${ }^{4}$ O. S. Balogun \\ ${ }^{1,2}$ Department of Mathematics, Modibbo Adama University of Technology, P.M.B 2076, Yola. Adamawa State, \\ Nigeria. \\ ${ }^{3}$ Department of Mathematics, Usmanu Danfodiyo University, Sokoto. \\ Sokoto State of Nigeria. \\ ${ }^{4}$ Department of Statistics and Operations Research, Modibbo Adama University of Technology, P.M.B 2076, \\ Yola. Adamawa State, Nigeria.
}

\begin{abstract}
This research deals with mathematical modelling of malaria transmission in North Senatorial Zone of Taraba State, Nigeria. The SIR proposed by Kermack and McKendrick and data obtained from Essential Programme on Immunisation (EPI) unit, F.M.C., Jalingo, Taraba state were used to analyse the rate of infection of malaria in the zone. From our analysis, we found out that the reproduction ratio $\left(R_{0}\right)>0$. Based on the reproduction ratio $R_{0}$, which is greater than 0, implies that the force of malaria infection in Taraba North Senatorial Zone is high. The researchers also make recommendations for the reduction of malaria in the zone.
\end{abstract}

Keywords: Malaria, stability, equilibrium states, epidemics.

\section{Introduction}

Malaria is a life-threatening disease widely spread in tropical and sub tropical regions, including Africa, Asia, Latin America, the Middle East and some parts of Europe. The most cases and deaths occur in Sub-Sahara Africa. In 2006 there were almost 250 million cases of malaria, causing nearly one million deaths (Rollback Malaria, 2010). However, malaria is preventable and curable. By making appropriate models for the spread of malaria one can understand the underlying process and develop effective prevention strategies.

Malaria is caused by parasites of the species plasmodium. The parasites are transmitted to humans through the bites of infectious female mosquito (vectors). The malaria parasite enters a human when an infectious mosquito bites a person. After entering a human the parasite transforms through a complicated life cycle. The parasites multiply in the human liver and blood-stream. Finally, when it has developed into an infectious form, it spreads the disease to a new mosquito that bites the infectious human. After approximately 10-15days the mosquito takes her next blood meal and can infect a new person. After a human gets bitten by symptoms appear in about 9 - 14 days (Rollback Malaria, 2010). The most common symptoms are headache, fever and vomiting. If the infected human does not get drugs the infection can progress and become lifethreatening. One of the most basic epidemiological models is the so called SIR model from Kermack and Mckendric ( 1927). This model is usually used to model the spread of a disease, not only the spread of malaria. The model describes the different states which a human can be in. The three states are Susceptible, Infectious and Recovered. A human moves through the different states at different rates.

Numerous researches have been done in the area of mathematical modeling of malaria. These begin in 1911 with Ross, who was awarded with a noble price for his work. His model was very simple and has been greatly extending during the year. Kermack and Mckendrick (1927) came up with the improved SIR model of epidemics.

Macdonald (1957) improved the model to a two dimensional model with one variable representing humans and one variable representing mosquito.

Several researches have been done on malaria, some relevant publications are the research done by Anderson and May (1991), Puntani and I-ming (2010), Hyun (2000), Isao et al., Jia (2008), Okosun (2010), Rafikov et al., Makinde and Okosun (2011). The increase in the epidemic in the sub-Africa regions has created much vacuum. It is in the light of filling the vacuum created that this research is carried out. This research attempts to look at mathematical modeling of malaria transmission in Taraba North senatorial zone of Taraba state. 


\section{Mathematical Model}

The SIR model proposed by Kermack and Mckendrick describes the different states which a human can be in; the three states are Susceptible, Infectious and Recovered. A human moves through the different states at different rates. Human enters the system in the susceptible state when born at the rate $B$. A susceptible human enters the infectious state at the rate $\beta$ when receiving the disease, from the infected state the human can either move to the recovered state at the rate $\alpha$ or the human can leave the system by death at the rate $d$. Human can also leave the susceptible, infected and recovered states due to natural death at rate $\mu$. The total population is denoted as $N$. The interaction between the states of this model is illustrated in figure 1 .

Model Diagram

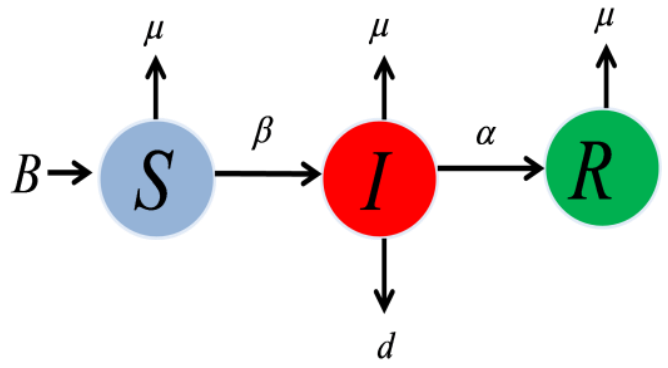

\section{Model Equations}

Figure 1: The basic SIR model with rates $B, \beta, \alpha, \mu$ and $d$ equation is:

This model can be described by a set of ordinary differential equations. The resulting system of

$$
\begin{aligned}
& \frac{d s}{d t}=B-\beta S I-\mu S \\
& \frac{d I}{d t}=\beta S I-I(\alpha+d+\mu) \\
& \frac{d R}{d t}=\alpha I-\mu R
\end{aligned}
$$

Assumptions of the SIR Model

* Birth rate and natural death rate are constant

* There is homogeneous interaction in the population

* The disease is spread by contact between parasites and the individual host

Existence of Equilibrium States of SIR Model

Here, we represent the results of the existence of equilibrium points of the SIR model.

\section{Equilibrium Analysis}

At equilibrium: $\frac{d S}{d t}=\frac{d I}{d t}=\frac{d R}{d t}=0$

Equations (1) - (3 becomes)

$$
\begin{aligned}
& B-\beta S I-\mu S=0 \\
& \beta S I-I(\alpha+d+\mu)=0 \\
& \alpha I-\mu R=0
\end{aligned}
$$

The incidence rate $(\mathrm{F})$ : in the above model, the function $F=\beta I S$ models the transition rate from the compartment of susceptible individuals to the compartment of infectious individuals. This is the incidence rate of malaria parasites infection on the population.

Here we consider incidence rate e.g infection that depends on the fraction of infectious since the population is large.

Thus, $F=\frac{\beta I S}{N}$

The basic reproduction number $\left(R_{0}\right)$ : 


$$
R_{0}=\frac{\beta}{(\alpha+\alpha+\mu)}
$$

This is the number of new infections from single infected in a population where all subjects are susceptible: the reproduction number strongly determines the rate of infection. From equation (2)

$$
\begin{aligned}
& \frac{d I}{d t}=\beta S I-I(\alpha+d+\mu) \\
& \frac{\beta}{(\alpha+d+\mu)}(\alpha+d+\mu) S I-I(\alpha+d+\mu) \\
& \frac{d I}{d t}=R_{0}(\alpha+d+\mu) S I-I(\alpha+d+\mu) \\
& \frac{d I}{d t}=\left[R_{0} S-1\right]-I(\alpha+d+\mu)
\end{aligned}
$$

It yield that for $R_{0}>0$ in the equation (7) above:

$\frac{d I}{d t}>0$

This means that there will be a proper malaria outbreak in the zone with an increase in the number of infected individuals in the population. The disease free equilibrium is unstable and the host will not recover from the disease.

Also, if $R_{0}<0$

$\frac{d I}{d t}<0$

In this case, the disease can never cause a proper epidemic outbreak. Thus, the human host will always recover from the disease.

From equation (5)

$[\beta S-(\alpha+d+\mu)] I=0$

$I=0$ or $\beta S-(\alpha+d+\mu)=0$

Sub $I=0$ into equation (6) we have,

$-\mu R=0 \Rightarrow R=0$

$\beta-\mu S=0$

$S=\beta / \mu$

Thus, the disease free equilibrium is:

$E_{0}=[B / \mu, 0,0]$

From equation (8) we have,

$$
\begin{aligned}
& \beta S-(\alpha+d+\mu)=0 \\
& S=\frac{(\alpha+d+\mu)}{\beta}
\end{aligned}
$$

Sub (11) into equation (4) we get,

$$
\begin{aligned}
& B-\beta\left[\frac{(\alpha+d+\mu)}{\beta}\right] I-\mu\left[\frac{(\alpha+d+\mu)}{\beta}\right]=0 \\
& B-(\alpha+d+\mu) I-\mu \frac{(\alpha+d+\mu)}{\beta}=0
\end{aligned}
$$


$(\alpha+d+\mu) I=B-\mu \frac{(\alpha+d+\mu)}{\beta}$

$(\alpha+d+\mu) I=\frac{\beta B-(\alpha+d+\mu)}{\beta}$

$I=\frac{\beta B-(\alpha+d+\mu)}{\beta(\alpha+d+\mu)}$

Sub (12) into equation (5) we get,

$\alpha I-\mu R=0$

$\alpha\left[\frac{\beta B-(\alpha+d+\mu)}{\beta(\alpha+d+\mu)}\right]-\mu R=0$

$R=\alpha\left[\frac{\beta B-\mu(\alpha+d+\mu)}{\beta \mu(\alpha+d+\mu)}\right]$

Thus, the epidemic equilibrium state is:

$E_{i}=\left[\frac{(\alpha+d+\mu)}{\beta}, \frac{\beta B-\mu(\alpha+d+\mu)}{\beta(\alpha+d+\mu)}, \frac{\beta B-\mu(\alpha+d+\mu)}{\beta \mu(\alpha+d+\mu)}\right]$

IV Stability Analysis

We established their stability of equilibrium points from the Jacobian matrix of equation (1) to (3) which is given as:

$J=\left[\begin{array}{ccc}-\beta I-\mu & -\beta S & 0 \\ \beta I & \beta S-(\alpha+d+\mu) & 0 \\ 0 & \alpha & -\mu\end{array}\right]$

$J=\left[\begin{array}{ccc}-\beta I-\mu-\lambda & -\beta S & 0 \\ \beta I & \beta S-(\alpha+d+\mu)-\lambda & 0 \\ 0 & \alpha & -\mu-\lambda\end{array}\right]$

Where $\lambda$ are the eigen values of $J$

$$
\begin{aligned}
& \operatorname{Det}(J)=(-\beta I-\mu-\lambda)\left|\begin{array}{cc}
\beta S-(\alpha+d+\mu)-\lambda & 0 \\
\alpha & -\mu-\lambda
\end{array}\right| \\
& =(-\beta I-\mu-\lambda)[(\beta S-(\alpha+d+\mu)-\lambda)(-\mu-\lambda)] \\
& =[-\mu-\lambda]\left[\left[\frac{\beta B}{\mu}-(\alpha+d+\mu)-\lambda\right][-\mu-\lambda)\right]
\end{aligned}
$$

Thus, the disease free equilibrium is stable when $\frac{\beta B}{\mu}<(\alpha+d+\mu)$ where $\frac{\beta B}{\mu}$ the number of is infected in the population.

\section{Results and Discussion}

Data collected from North Senatorial Zone of Taraba State Nigeria: Table 1 shows the statically analysis of the zone projected population for 2011 and Table 2 shows reported cases of Malaria from 2007 to 2011 in Taraba state. Figure 1 to 4 narrates the basic reproduction ratio in

\begin{tabular}{|l|l|l|l|}
\hline $\begin{array}{l}\text { Local } \\
\text { Government }\end{array}$ & Population & \\
\hline Ardo-Kola & & 96281 & \\
\hline Jalingo & & 160303 & \\
\hline
\end{tabular}


Mathematical Modelling Of Malaria Transmission In North Senatorial Zone Of Taraba State Nigeria

\begin{tabular}{|l|l|l|l|}
\hline K/Lamido & & 219205 & \\
\hline Lau & & 108929 & \\
\hline Yorro & & 103929 & \\
\hline Zing & & 150065 & \\
\hline Total & & $\mathbf{7 9 3 6 4 3}$ & \\
\hline
\end{tabular}

Table 1: Taraba North Senatorial zone projected population 2011

\begin{tabular}{|c|c|c|}
\hline Years & Cases & $\mathbf{( 2 0 0 7 - 2 0 1 1 )} \%$ \\
\hline 2007 & 140,247 & 22 \\
\hline 2008 & 160,420 & 25 \\
\hline 2009 & 117,853 & 17 \\
\hline 2010 & 198,867 & 32 \\
\hline 2011 & 176,009 & 28 \\
\hline
\end{tabular}

Table 2: Malaria cases in Taraba State (2007-2011)

Calculation of the basic reproduction ratio with target population, 2011:

From equation 7 ,

$$
\begin{aligned}
& R_{0} S-1=0 \\
& R_{0} * S=1 \\
& R_{0}=\frac{1}{S} \\
& R_{0} \alpha \frac{K}{S(t)}
\end{aligned}
$$

For all $K>0$

1. $R_{0}$ for Ardo-Kola

$$
R_{0}=\frac{K}{\text { Population }}=0.0000103863 k
$$

For all $K=0.25,0.5,0.75,1$

$R_{0}=0.0000025966,0.00000519315,0.0000077895,0.0000103863$

2. $R_{0}$ for Jalingo

$$
R_{0}=\frac{K}{\text { Population }}=0.00000623818 k
$$

For all $K=0.25,0.5,0.75,1$

$R_{0}=0.0000015596,0.000000311909,0.00000046786,0.00000623898$

3. $R_{0}$ for $\mathrm{K} /$ Lamido

$$
R_{0}=\frac{K}{\text { Population }}=0.000000456193 k
$$

For all $K=0.25,0.5,0.75,1$

$R_{0}=0.00000114048,0.00000228097,0.00000342145,0.00000456193$

4. $R_{0}$ for Lau

$$
R_{0}=\frac{K}{\text { Population }}=0.00000918029 k
$$

For all $K=0.25,0.5,0.75,1$

$R_{0}=0.00000229507,0.000000459015,0.00000068852175,0.00000918029$

5. $R_{0}$ for Yorro 

$R_{0}=\frac{K}{\text { Population }}=0.00000962195 k$

For all $K=0.25,0.5,0.75,1$

$R_{0}=0.00000240549,0.000000480975,0.000000721647,0.00000962195$

6. $R_{0}$ for Zing

$$
R_{0}=\frac{K}{\text { Population }}=0.00000666377 \mathrm{k}
$$

For all $K=0.25,0.5,0.75,1$

$R_{0}=0.00000166595,0.000000333189,0.000000497828,0.00000666377$

Hence the basic reproduction ratio $R_{0}$ for Taraba North Senatorial zone is given as

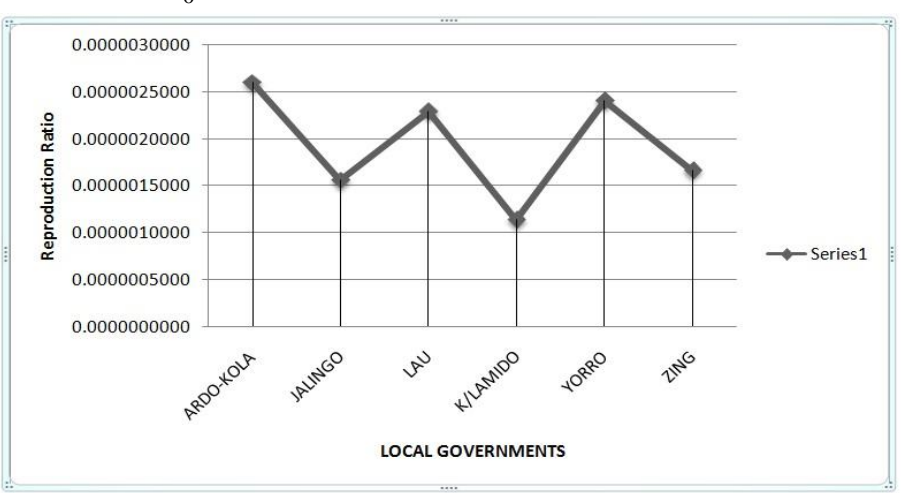

Figure 1 above: Reproductive Ration at $\mathbf{K}=\mathbf{0 . 2 5}$

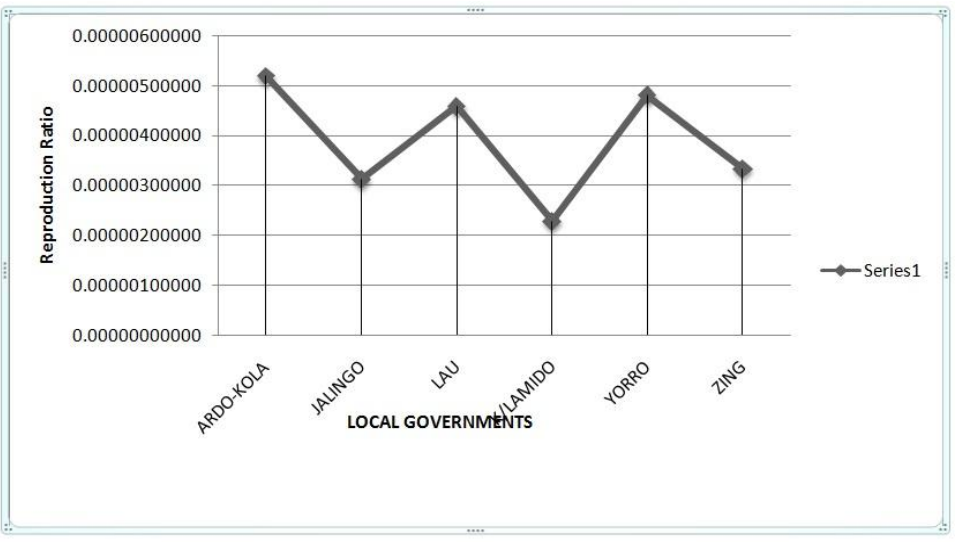

Figure 2 above: Reproductive Ration at $\mathrm{K}=0.50$

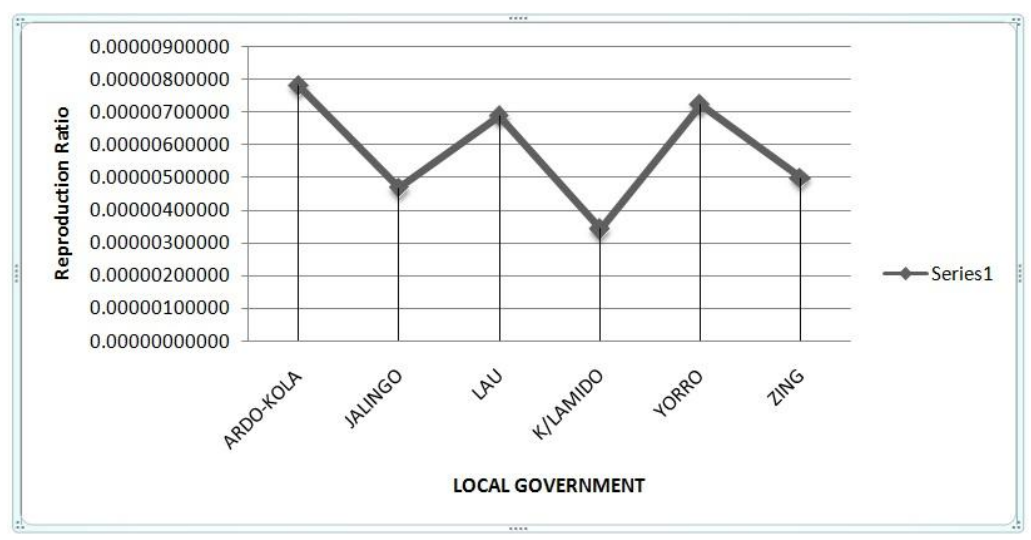

Figure 3 above: Reproductive Ration at $\mathbf{K}=\mathbf{0 . 7 5}$ 


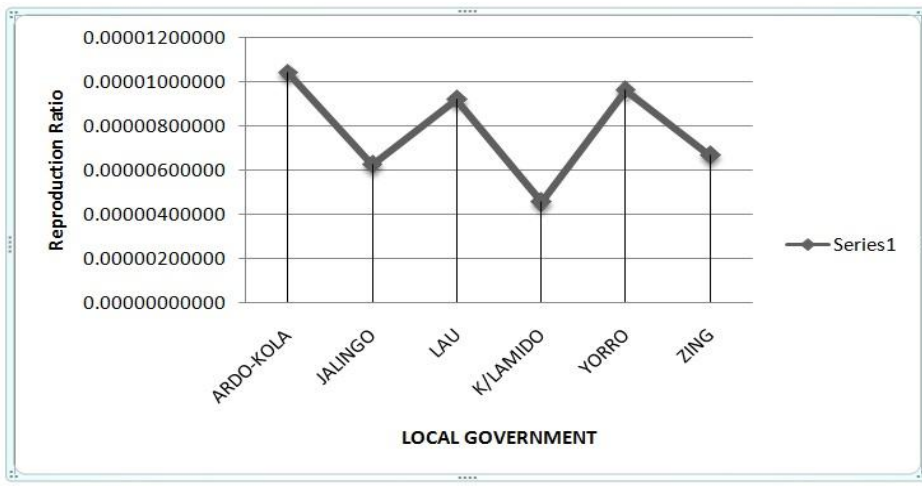

Figure 4 above: Reproductive Ration at $\mathbf{K}=\mathbf{1 . 0}$

\section{Interpretation of the result}

* The force of infection increase over the years and this makes the number of susceptible individuals in the population to decrease. This validates the relation $S \alpha \frac{K}{R_{0}}$

* The number of new infections increases over the years in this population of which every individual is susceptible and thus the basic reproduction ratio is positive i.e $\left(R_{0}>0\right)$. Therefore, there will be malaria outbreak in the zone with an increase in the number of the infected individuals in the zone.

* The number of infected individuals keep increasing from 2007 to 2011 satisfying the assertion that as $t \rightarrow \infty, S(t) \rightarrow 0$ and $I(t) \rightarrow N$.

\section{Conclusion}

Conclusively, we will like to table the following to help reduce the rate of infection of malaria in Taraba North senatorial zone.

$\checkmark$ Malaria is a major cause of morbidity and mortality in Nigeria and Taraba North Senatorial zone. Hence the need to address the menace.

$\checkmark \quad$ There should be early treatment of malaria

\section{Recommendations}

In order to eradicate malaria from Taraba North Senatorial zone, the following recommendations should be considered:

1) The public should take adequate step towards preventing themselves from malaria attack.

2) The public should remove potential mosquito breeding sites like stagnant water to reduce number of mosquito.

3) The government should provide clean environment and drainage system for the inhabitants of Taraba North Senatorial zone.

4) Vaccines against fever should be made available by government

\section{References}

[1] Anderson, R. M., May, R. M. (1991). Infectious Diseases of Humans: Dynamics and Control.Oxford University Press, Oxford.

[2] Hyun, M. Y. (2000). Malaria transmission model for different levels of acquired immunity and temperature dependent parameters vector. Rev. Saude Publica., 34(3): 223-231.

[3] Isao, K., Akira, S., Motoyoshi, M. (2004). Combining Zooprophylaxis and insecticide spraying A malaria-control strategy limiting the development of insecticides resistance in vector mosquitoes. Proc. R. Soc. Lond., 271: 301-309.

[4] Jia, L. (2008). A malaria model with partial immunity in humans. Math. Bios. Eng., 5(4): 789- 801.

[5] Kermack, W. O. and Mckendric, A.G. ( 1927). A contribution to the mathematical theory of epidemics: preceedings of the Royal society of London. Series A, Containing papers of a mathematical and physical character, 115:700-721.

[6] Makinde, O. D., Okosun, K. O. (2011). Impact of chemo-theraphy on optimal control of malaria disease with infected immigrants. BioSystems. 104:32-41

[7] McDonald, G. (1957) The epidemiology control of malaria, Oxford university press, London.

[8] Okosun, K. O. (2010). Mathematical epidemiology of Malaria Disease Transmission and its Optimal Control Analyses, Ph.D. thesis, University of the Western Cape, South Africa Puntani, P. I-ming, T. (2010). Impact of cross-border migration on disease epidemics: case of the P. falciparum and P. vivax malaria epidemic along the Thai-Myanmar border. J. Bio. Sys., 18(1): 55-73

[9] Rafikov, M., Bevilacqua, L., Wyse, A. A. P. (2009). Optimal control strategy of malaria vector using genetically modified mosquitoes. J. Theore. Bio., 258: 418-425.

[10] RollbackMalaria.What is Malaria?http://woo.rollback-malaria.org/cmc$10)$.

upload/0/000/015/372/RBMinfosheet-1.pdf.(2010-05-

[11] Ross, R. (1911). The prevention of malaria John Murray, London, 1911. 